\title{
Molecular Crosstalk amongst Anti-Orexigenic Ghrelin and Adenosine Monophosphate-Activated
}

\author{
Akanksha Singh* \\ Rashtrasant Tukadoji Maharaj Nagpur University, Nagpur, India \\ *Corresponding Author: Akanksha Singh, Rashtrasant Tukadoji Maharaj Nagpur \\ University, Nagpur, India.
}

Received: July 12, 2021

Published: July 26, 2021

(C) All rights are reserved by Akanksha Singh.

\begin{abstract}
Ghrelin has proved to be a crucial mediator in stimulation of obesity. It elevates appetite by eliminating the action of GHS-R1a of neurological NPY/AgRP thus enhancing orexigenic activity. Such negative effects had been neutralized by the production of auto-ghrelin antibodies which are self-catalytic neutralizing the obese conditions by directly targeting AMPK active site which is known to stimulate appetite. Suppression of AMPK thus playing a central role might stimulate the activation of GHS-R1a thus leading to controlled release of ghrelin hormone. In this study, when mice were fed with high calorie diet exposed to the anti- ghrelin antibodies prepared by ELISA technique, after few hours, anti- ghrelin showed maximum activity thus decreasing ghrelin levels originally present. The study mainly focusses on the determining the interaction occurring between obese mediated ghrelin molecule and AMPK.
\end{abstract}

Keywords: Ghrelin; GHS-R1a; NPY/AgRP; Orexigenic; AMPK; ELISA; Obesity

\section{Introduction}

Obesity is a multifactorial disorder affecting humans especially younger adults at a global level. According to World Obesity Report 2021 describing the impact of obesity in the times of pandemic, larger number of deaths that occurred due to COVID-19 comprises the population that were obese [1]. Obesity is directly proportional to expenditure of ATP done by the cells of the body. Many models have been proposed till date to visualize the pattern by which the body performs functions at the expense of large amount of ATP [2]. Proteomic biomarkers such as neuropeptide $Y$, orexins and ghrelin are known to play the role in obesity. Ghrelin has gained much importance due to its ability to elevate the appetite to higher levels. It has been found that the obese hormone ghrelin although secreted at lower levels in the individuals affected by obesity but elevates the appetite. This orexigenic marker induces the food appetite at a rate of $30 \%$. While the ghrelin is present at sufficient levels in the circulatory system, it send signals to amygdala in the brain that contains GHS-R1a [3]. High levels of ghrelin will ultimately block the activity of the GHS-R1a that is responsible for controlling the food appetite thus maintaining orexigenicity.
The ghrelin that is acylated at the rate of $10 \%$ only binds to GHS$\mathrm{R} 1 \mathrm{a}$ receptor in the brain and rest $90 \%$ remains un-acylated [4]. It is crucial to nullify the cons produced due to reduced activity of GHS-R1a in the amygdala by generation of anti-ghrelin antibodies. Lipocyte cells obtained were cultured and anti-ghrelin antibodies were produced by rDNA technology. The anti-ghrelin antibodies were quantified and detected by the ELISA technique. The antighrelin antibodies were intravenously injected to the mouse previously fed with high calorie diet. After a week, the blood was taken from the mouse and antibody concentration was determined. The study determined the interaction that might occur at the genetic level amongst the obese hormone ghrelin and the AMPK which is the important marker on the surface of GHSR1-a.

Neuromolecule AMPK and its interaction with AICAR molecule

AMPK is one of those neuro-molecules that are hugely important in controlling food appetite and other related functions. At the times of fasting, the activity of AMPK is elevated at high levels. AICAR (5-aminoimidazole-4-carboxamide 1 B- D-ribofuranoside) that acts as a precursor of the activator AMPK stimulates the inter- 
action with the food hormone ghrelin. Marmots were previously exposed to AICAR at optimum conditions were known to indicate increased appetite [5]. On the contrary, the marmots that were just fed with the saline water didn't indicate any significant results [6]. This AICAR molecule directly binds to the $\alpha$ and $\beta$ subunit of AMPK molecule. The molecular binding between the AICAR and AMPK involves the initial attachment of the AICAR molecule to the adenine transporters mainly catalysed by the adenosine kinase. This follows the secretion of intermediate named ZMP from purine synthesis pathway that usually accumulates within the cell at a concentration in milli molar [7]. The ZMP molecule is mainly activated by enzyme AICAR transformylase. This activated ZMP will now be responsible for the active functioning of AMPK biomolecule.

\section{Activated AMPK accelerates interaction with Ghrelin}

The AMP molecule previously activated is conjugated to several molecules of AMP and ATP thus enhancing phosphorylation activity [8]. These conditions lead to generation of the food appetite. Soon after the activated AMPK shuts down cycle leading to ATP production, Ghrelin already present at high levels stimulate the secretion of biomolecules such as orexigenors such as NPY and AgRP by interaction with GHSR subunit that ultimately allows the influx of calcium molecules. Further, the binding of calcium with the calmodulin mediated protein kinase undergoes the phosphorylation of AMPK subunit. Ghrelin when administered in the mice at regular intervals in the Intracerebroventricular system was known to elevate appetite [9]. It was then hypothesized that AMPK might be phosphorylated at high levels that lead to excess production of the hormone thus contributing obesity.

\section{Conclusion}

Obesity is measured in terms of how much of energy expense is done by the human body. Ghrelin is known as food hormone that is known to increase appetite at high levels usually after fasting. This hormone has greater affinity for the GHS-R1a located in the amygdala region. At the same time, it also interacts effectively with the AMPK molecule. AMPK molecule is activated by AICAR molecule and followed by the activation of $\mathrm{Ca}^{2+}$ stores. This further enhances the activity of GHS-R1a that when binds to the hormone will lower its production. There is need to study the rare biomarkers that may inhibit the ghrelin activity in the greater detail.

\section{Conflicts of Interest}

There are no conflicts of interest related to the study.

\section{Acknowledgements}

This work has been supported by Rashtrasant Tukadoji Maharaj Nagpur University, Nagpur and CSIR-National Environmental Engineering Research Institute, Nagpur.

\section{Bibliography}

1. '2722_WOF_-_COVID-19_and_Obesity-The_2021_Atlas_WEB. pdf' (no date) (2021).

2. Trayhurn P. "The biology of obesity". The Proceedings of the Nutrition Society 64.1 (2005): 31-38.

3. Alvarez-Crespo M., et al. "The amygdala as a neurobiological target for ghrelin in rats: neuroanatomical, electrophysiological and behavioral evidence". PloS one 7.10 (2012): e46321.

4. Delporte C. "Structure and physiological actions of ghrelin". Scientifica (2013): 518909.

5. Lim CT., et al. "AMPK as a mediator of hormonal signalling". Journal of Molecular Endocrinology 44.2 (2010): 87.

6. Florant GL., et al. "To eat or not to eat: the effect of AICAR on food intake regulation in yellow-bellied marmots (Marmota flaviventris)". Journal of Experimental Biology 213.12 (2010): 2031-2037.

7. Kim J., et al. "AMPK activators: mechanisms of action and physiological activities". Experimental and Molecular Medicine 48.4 (2016): e224-e224.

8. Jeon SM. "Regulation and function of AMPK in physiology and diseases". Experimental and Molecular Medicine 48.7 (2016): e245-e245.

9. Huynh MKQ., et al. "Hypothalamic AMPK as a regulator of energy homeostasis". Neural Plasticity (2016).

\section{Volume 3 Issue 8 August 2021 (C) All rights are reserved by Akanksha Singh.}

